\title{
Ihre kostenlose Kleinanzeige
}

Suchen Sie Praxisvertreter oder Kollegen, mit denen Sie zusammenarbeiten wollen? Haben Sie Medizingeräte, Praxismobiliar etc. abzugeben oder sind Sie selbst an Entsprechendem interessiert? Nutzen Sie unser Serviceangebot und schalten Sie eine kostenlose "Kleinanzeige“. Senden oder faxen Sie uns den ausgefüllten Coupon. In der nächsten erreichbaren Ausgabe der HNO-Nachrichten werden wir Ihre eigene Wunschanzeige veröffentlichen.

Biete

Verkaufe 2 Johnson Instrumentenschränke und 1 Zeiss OPMI 9 mit Wandhalterung

E-mail: praxis@hnogiessen.de 용 064134032

laC-Hörprüfkabine Modell Mini 250 Industrial Acoustics Company Baujahr 1990

sehr gut erhalten, 1000 Euro

E-Mail: mail@hno-langen.de

西 01723060935
Hörkabine und Audiometer

der Fa. Hartmann zu verkaufen

\% 087313266640

Verkaufe Otopront „Behandlungseinheit Ravensburg" sowie Instrumentenschrank „Wiesbaden" mit Operationsmikroskop "Zeiss Opmi 9" in optisch und technisch einwandfreiem Zustand sowie Erbe Koagulationsgerät mit Argonplasmaergänzung APC 300 inklusive Wagen,

Preis Verhandlungssache

范 0722125788

\section{Suche}

HNO-Arzt in GP sucht Nachfolger PLZ 66

空 0177296567

HNO-Gemeinschaftspraxis Nähe Tübingen sucht Nachfolger 莤 01739396311

Suche Nachfolger für gutgehende HNO-Praxis in Trier, amb. Op. und Belegbetten 落 065175357

\section{Bestellschein}

Bitte deutlich und mit Druckbuchstaben ausfüllen und Telefonnummer, Faxnummer oder E-Mail-Adresse angeben!

Bitte veröffentlichen Sie für mich kostenlos folgenden Anzeigentext in einer der nächsten Ausgaben der HNO-NACHRICHTEN:
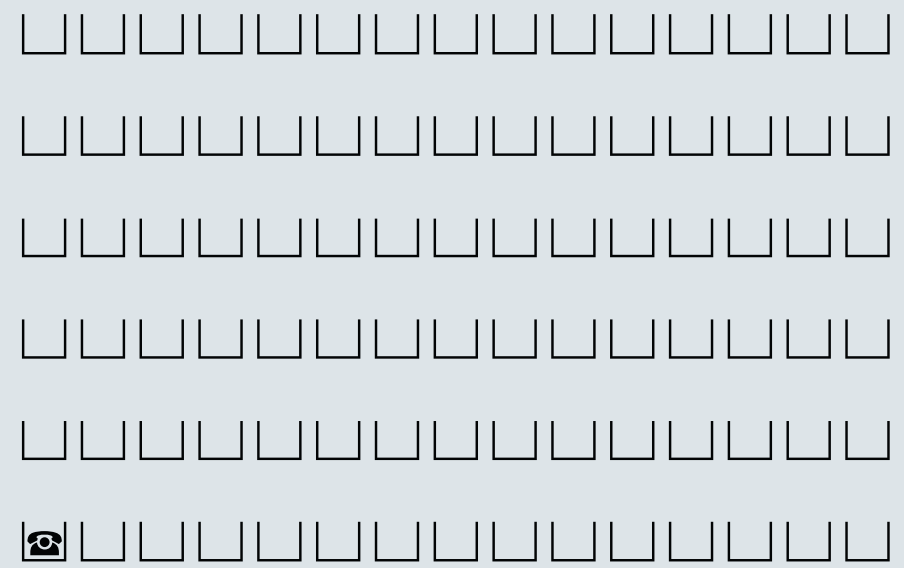

Springer Medizin, Urban \& Vogel GmbH Redaktion HNO-NACHRICHTEN, Aschauer Str. 30 81549 München Fax 0892030 43-14 00 E-Mail: gabriele.zoerrgiebel @springer.com$$
\text { (aspringercom }
$$

Name, Vorname

Geburtsdatum

Straße, Nr.

$\overline{\mathrm{PLZ}, \text { Ort }}$

$\overline{\text { Datum }}$

Unterschrift 